\title{
The Influence of the Temperature on Positron Annihilation Lifetime Spectra in Porous Sol-Gel Glass Doped with Ag Nanoparticles
}

\author{
M. Gorgol ${ }^{a, *}$, B. JAsińska ${ }^{a}$, R. Reisfeld ${ }^{b}$ AND V. LEVChenKo ${ }^{b}$ \\ ${ }^{a}$ Institute of Physics, Maria Curie-Skłodowska University, pl. M. Curie-Skłodowskiej 1, 20-031 Lublin, Poland \\ ${ }^{b}$ Chemistry Institute, Hebrew University of Jerusalem, Givat-Ram, Jerusalem 91904, Israel
}

\begin{abstract}
Silver nanoparticles with continuous size distribution from 10 to $20 \mathrm{~nm}$ were incorporated into porous glass produced using sol-gel technique. Positron annihilation lifetime spectroscopy was used to compare obtained material with an undoped reference glass. The positron annihilation lifetime spectroscopy measurement were made in a broad temperature range (from $-180^{\circ} \mathrm{C}$ to $200^{\circ} \mathrm{C}$ ). The experimental lifetime values were compared with the predictions of the extended Tao-Eldrup model of the temperature range. Experimental results for the reference material were in agreement with the model, except for a small discrepancy at low temperatures. In doped material substantial changes and non-monotonous temperature dependences of intensity and lifetime values were observed. Heating the material to $200{ }^{\circ} \mathrm{C}$ resulted in reorganization of the material structure.
\end{abstract}

DOI: $10.12693 /$ APhysPolA.125.778

PACS: 78.70.Bj, 36.10.Dr, 65.60.+a, 61.43.Gt

\section{Introduction}

Silver nanoparticles (Ag NPs) have become interesting material due to their possible practical applications e.g. in medicine. Therefore, conducting research of newly synthesized materials containing Ag NPs is very important. A sol-gel technique, in which all dopant molecules are added into a solution as a stage of material preparation, is not expensive and commonly used for obtaining the porous materials with various properties.

Positron annihilation lifetime spectroscopy (PALS) can give information about the internal structure of porous materials and the change of their behavior as a function of various factors such as pressure [1], or temperature $[2-5]$. Two parameters, lifetime $(\tau)$ and intensity $(I)$, can be ascribed to specific components of the PALS spectra during the analysis. In simplified interpretation, the intensity can give information about the number of free volumes, presence of various chemical groups and bonds inside the material. The ortho-positronium (o-Ps) lifetime values give information on the free volumes size. The $\tau$ values, corresponding to the $o$-Ps annihilation inside the mesopores, can be used to determine the pore size distribution with the use of the extended Tao-Eldrup (ETE) model [6]. In this model shape of pore is approximated by the potential well, where $o$-Ps can occupy either ground or excited energy levels. Due to the population of the states the model predicts decrease of $o$-Ps lifetime with the increase of temperature, even if the size of the pores are not changed.

*corresponding author; e-mail: marek.gorgol@gmail.com

\section{Experimental}

The examined materials were prepared by the sol-gel procedure, obtained by hydrolysis and polycondensation of the ester of silicic acid in alcoholic solvent. The result was a solid non-organic glass. In the case of doped material, Ag NPs were added, at required concentration. The procedure of material preparation was described in [7]. Silver NPs were obtained using procedure described in [8].

The sample, surrounding the ${ }^{22} \mathrm{Na}$ source, with the activity of $5 \mu \mathrm{C}$, was placed in a vacuum chamber. A turbomolecular pump provided the pressure of $10^{-5} \mathrm{~Pa}$. Initially each sample was degassed for $24 \mathrm{~h}$, at a room temperature.

Apparatus, which allowed conducting measurements in a wide temperature range, consisted of the resistance heater and the dewar vessel with liquid nitrogen. The temperature was controlled by the Shimaden FP-21 PID controller. Each sample was cooled down from the room temperature to the $-180^{\circ} \mathrm{C}$, then heated up to $200^{\circ} \mathrm{C}$ and then cooled down again to the room temperature.

Positron annihilation lifetime spectra were collected using standard fast-slow delayed coincidence spectrometer, equipped with two $\mathrm{BaF}_{2}$ scintillators $\left(\varnothing 1 \times 1.5^{\prime \prime}\right.$ in the START signal branch and $\varnothing 1 \times 1.5^{\prime \prime}$ in the STOP signal branch). The setup was adapted to do measurement in a whole range of lifetimes up to 142 ns. Therefore, time range of the time-to-amplitude converter was $2 \mu \mathrm{s}$, while the time width of the single channel of multichannel analyzer was 116.3 ps. The STOP energy window was widely open in order to collect the low energy gamma quanta from three gamma annihilation of $o-\mathrm{Ps}$. The resolution curve was approximated using two Gaussians: one with a FWHM of about $300 \mathrm{ps}$ with the contribution of $80 \%$, and the other with FWHM of about 700 ps and contribution of $20 \%$. 
The number of positron registration was about $1.7 \times$ $10^{6}$ counts per hour, and total count number of spectrum at each temperature value was $5 \times 10^{6}$.

The lifetime spectra were analyzed with using two different approaches:

1. Convolution with the use of LT 9.2 program [9],

2. Deconvolution obtained by MELT program [10].

The pore sizes calculations were conducted with the use of EELVIS [11] program, based on the ETE model assumptions. Because of the most probable shape of pores, obtained in sol-gel technique, the spherical potential well was assumed as a pore shape approximation in the ETE model.

\section{Results and discussion}

The LT analyses have been conducted using four components with the time dispersion of the longest-lived component. Particular components were ascribed to $p$-Ps (fixed at $125 \mathrm{ps}$ ), free annihilation of $\mathrm{e}^{+}$and $\mathrm{e}^{-}$and two components connected to $o$-Ps annihilation in micropores and mesopores.

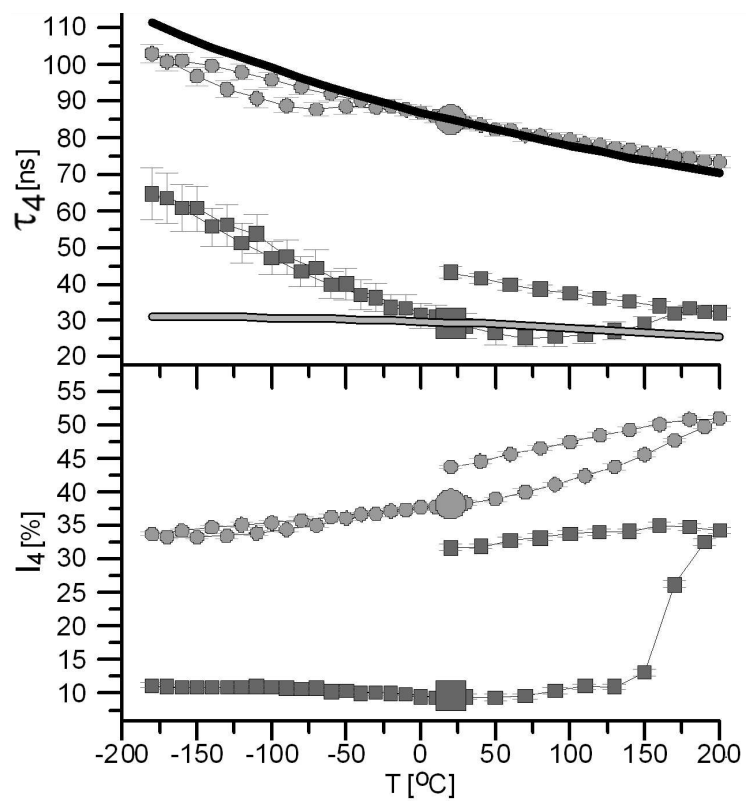

Fig. 1. The $o$-Ps lifetime and intensity of longest-lived component versus temperature. Symbols denote: circles - reference sol-gel glass, squares - glass doped with Ag nanoparticles. Initial values are highlighted with enlarged points. Fitted curves show the approximations of the ETE model.

Temperature dependences of the $\tau$ and $I$ values of the longest-lived component $\left(\tau_{4}, I_{4}\right)$ for the base material and doped with Ag NPs material were presented in Fig. 1. The initial values for the base material were $I_{4-0}=38.2(4) \%$ and $\tau_{4-0}=84.9(16)$ ns. This last value was used to calculate the mean pore size, and a value of $D_{0}=7.7(3) \mathrm{nm}$ was received. Lifetime values increased during cooling to the $-180^{\circ} \mathrm{C}$. At this temperature the $\tau_{4}$ reached the value of $102.9(27)$ ns, while in the prediction of the ETE model, this value should be 111.3 ns. During the heating the $\tau$ values were much more discrepant from the predictions of the model and a small hysteresis was visible. Above the $-50^{\circ} \mathrm{C}$ quite good agreement between the experimental and theoretical lifetime values can be observed. At $200^{\circ} \mathrm{C}$ the difference between the two values (measured and calculated from the ETE model) is $3.1 \mathrm{~nm}$ and experimental values are higher than theoretical ones. This could be explained as an effect of the thermal expansion of the material. An increase of longest-lived component intensity with temperature is similar to those previously observed for doped silicas $[12,13]$. After heating up to $200{ }^{\circ} \mathrm{C}$ higher $I_{4}$ values are observed. Such tendency, observed before for some photonic glasses [14], can be the result of breaking of the hydrogen bonds inside the material.

Doping a glass with Ag NPs resulted in large decrease of $I_{4}$ and $\tau_{4}(9.6(4) \%$ and $29.5(33) \mathrm{ns}$, respectively, at room temperature). Bigger errors found during spectra processing using LT program, in comparison to the base material, are the result of low intensity of $o$-Ps. The ETE calculation gave unreliable pores size of $2.51(8) \mathrm{nm}$. The great disagreement between experimental and theoretical results in whole temperature range was also observed. It suggests that the decrease of $I$ and $\tau$ values is not only connected with pores size reduction in the doped material, but also with the interaction of metallic nanoparticles with positrons or positronium. The possible interpretations of $\tau_{4}$ decrease could be the $o$-Ps quenching, while the $I_{4}$ decrease could correspond to free positrons trapping before the positronium formation. From $150^{\circ} \mathrm{C}$ to $200^{\circ} \mathrm{C}$ significant increase of both lifetime and intensity is observed. Moreover, these parameters do not return to their initial values after cooling down to the room temperature, which suggests the reorganization of the material structure. It is possible that during heating, nanoparticles migrated inside the material and created large NPs clusters. It could result in a decrease of the effects, corresponding to the $\tau$ and $I$ values reduction.

In order to check the influence of Ag NPs incorporation on lifetime spectra, deconvolution using MELT was done.

The lifetime distribution, received during spectra processing, is presented in Fig. 2. The sufficient statistics for MELT calculation required summing the spectra for $20^{\circ} \mathrm{C}$ and $0{ }^{\circ} \mathrm{C}$. Because of wider distribution and numerical procedures implemented into the program, an unreliable number of components was received. However, changes of lifetime distribution due to doping the material, or changes of the temperature can be observed.

In order to compare the results from LT and MELT, values of particular components obtained from these two methods of calculations are presented in Table. High value and wide distribution of $I_{4}$ corresponds to the existence of large amount of mesopores inside the base material. It is also confirmed by large proportion of the 


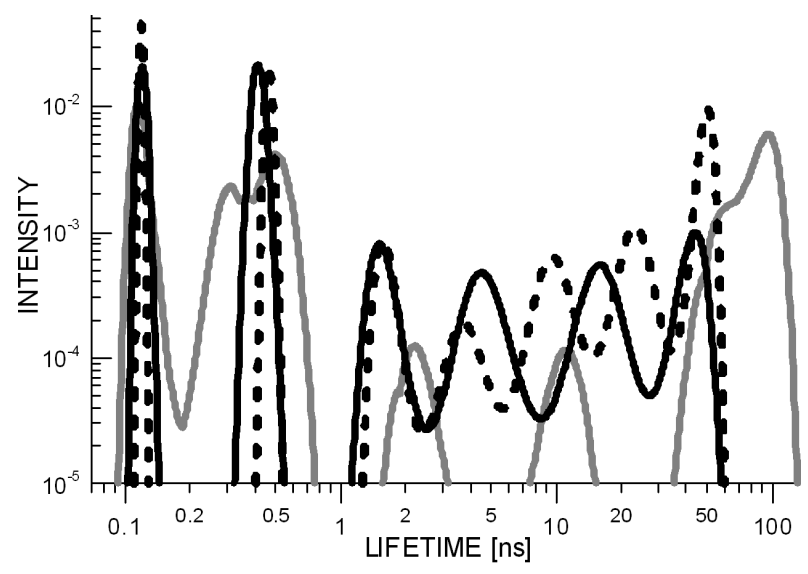

Fig. 2. The $o$-Ps lifetime distribution at room temperature received using MELT deconvolution. Symbols denote: grey curves - the reference material, black curves - glass incorporated with $\mathrm{Ag}$ nanoparticles, dashed curves - glass incorporated with $\mathrm{Ag}$ nanoparticles after heating the sample up to $200{ }^{\circ} \mathrm{C}$ and re-cooling to the room temperature.

TABLE

Lifetime and intensity values for all components at the room temperature received using two various programs for analyses: LT and MELT. Columns marked with * present values for the glass doped with Ag NPs after heating the sample up to $200{ }^{\circ} \mathrm{C}$ and re-cooling to the room temperature. Intensities $(I)$ and lifetimes $(\tau)$ of the multiplied peaks obtained by MELT were assigned to corresponding LT components.

\begin{tabular}{|c|c|c|c|c|}
\hline \multicolumn{5}{|c|}{ Base material } \\
\hline \multirow{2}{*}{$\begin{array}{l}\text { Number of component } \\
\text { obtained from LT }\end{array}$} & \multicolumn{2}{|c|}{ LT } & \multicolumn{2}{|c|}{ MELT } \\
\hline & $I[\%]$ & $\tau[\mathrm{ns}]$ & I [\%] & $\tau$ [ns] \\
\hline I & $31.9(5)$ & 0.125 & $26.4(15)$ & 0.118 \\
\hline \multirow{3}{*}{ II } & \multirow{3}{*}{$28.9(5)$} & \multirow{3}{*}{$0.468(45)$} & $9.3(57)$ & 0.294 \\
\hline & & & $4.2(86)$ & 0.371 \\
\hline & & & $21.2(58)$ & 0.504 \\
\hline \multirow{3}{*}{ III } & \multirow{3}{*}{$1.02(14)$} & \multirow{3}{*}{$3.8(4)$} & $0.1(12)$ & 1.7 \\
\hline & & & $0.6(11)$ & 2.3 \\
\hline & & & $0.7(3)$ & 11.0 \\
\hline IV & $38.2(4)$ & $84.9(16)$ & $37.6(2)$ & 85.2 \\
\hline \multicolumn{5}{|c|}{ Glass with Ag NPs } \\
\hline \multirow{2}{*}{$\begin{array}{l}\text { Number of component } \\
\text { obtained from LT }\end{array}$} & \multicolumn{2}{|c|}{ LT } & \multicolumn{2}{|c|}{ MELT } \\
\hline & I [\%] & $\tau[\mathrm{ns}]$ & I [\%] & $\tau[\mathrm{ns}]$ \\
\hline $\mathrm{I}$ & $35.3(8)$ & 0.125 & $33.4(4)$ & 0.120 \\
\hline II & $50.6(7)$ & $0.433(47)$ & $51.6(5)$ & 0.415 \\
\hline \multirow[t]{2}{*}{ III } & \multirow{2}{*}{$4.5(3)$} & \multirow{2}{*}{$2.55(20)$} & $3.5(10)$ & 1.576 \\
\hline & & & $3.4(15)$ & 4.68 \\
\hline \multirow{2}{*}{ IV } & \multirow{2}{*}{$9.6(4)$} & \multirow{2}{*}{$29.5(33)$} & $3.8(17)$ & 16.156 \\
\hline & & & $4.3(13)$ & 42.769 \\
\hline \multicolumn{5}{|c|}{ Glass with $\mathrm{Ag}$ NPs * } \\
\hline \multirow{2}{*}{$\begin{array}{c}\text { Number of component } \\
\text { obtained from LT }\end{array}$} & \multicolumn{2}{|c|}{ LT } & \multicolumn{2}{|c|}{ MELT } \\
\hline & I [\%] & $\tau[\mathrm{ns}]$ & I [\%] & $\tau[\mathrm{ns}]$ \\
\hline $\mathrm{I}$ & $34.8(5)$ & 0.125 & $32.9(2)$ & 0.119 \\
\hline II & $28.4(5)$ & $0.510(56)$ & $29.1(2)$ & 0.464 \\
\hline \multirow{3}{*}{ III } & \multirow{3}{*}{$5.21(25)$} & \multirow{3}{*}{$4.8(3)$} & $2.9(15)$ & 1.623 \\
\hline & & & $1.2(21)$ & 3.807 \\
\hline & & & $4.0(29)$ & 9.782 \\
\hline \multirow[t]{2}{*}{ IV } & \multirow[t]{2}{*}{$31.6(39)$} & \multirow[t]{2}{*}{$43.3(16)$} & 6.1(35) & 23.264 \\
\hline & & & $23.8(30)$ & 49.285 \\
\hline
\end{tabular}

longest-lived component (with very small intensity of the shorter $o$-Ps components) in the MELT results. The presence of broad and fragmented peak corresponding to the free annihilation component reflects a complicated electron structure of the material. After doping the material with Ag NPs the total lifetime and intensity values of $o$-Ps components significantly decreased and the lifetime distribution became broad. A significant decrease of $I_{4}$ and slight increase of $I_{3}$ is visible. Similar effect, observed in SBA-15 silica, was interpreted as a result of $o$-Ps migration from smaller to larger free volumes [15]. Due to lifetime shortening, the probability of $o$-Ps localization inside smaller free volumes increased. However, this interpretation could not be accurate for the sol-gel glass because of different material structure, especially that pores are closed. The increase of $p$-Ps intensity (of about $6.5 \%$ ) could be interpreted as a result of $o$-Ps to $p$-Ps conversion due to chemical activity (e.g. presence of active groups or bonds inside the molecules) of doped material. Additionally, a significant narrowing (with an increase of total intensity) of the peak corresponding to a free positron annihilation is visible in the MELT results. It would confirm the thesis that presence of metal nanoparticles results in trapping of positrons before the positronium formation.

After heating the doped sample to $200{ }^{\circ} \mathrm{C}$ and cooling it down, to room temperature, the increase of o-Ps component intensities was observed, while the intensity of component ascribed to free positron annihilation decreased. However, the shape of the peak corresponding to the second measurement cycle remained the same as in the doped material before heating. It suggests that the presence of nanoparticles still affected the lifetime spectra, but the probability of positron trapping decreased. It could also be the result of the AG NPs clusters formation, which can decrease the probability of appearance of positron in the vicinity of the electron density derived from the nanoparticles.

Comparison of $I_{4}$ and $\tau_{4}$ temperature dependences of the first and second measurement series for glass doped with Ag NPs was presented in Fig. 3. It can be seen that after material structure reorganization caused by heating up to $200^{\circ} \mathrm{C}$, the monotonous (and close to those usually observed) dependences for both lifetime and intensity were visible. It suggests that $\mathrm{Ag} \mathrm{NPs}$ cluster formation prevented further migration of nanoparticles. Discrepancy between experimental and theoretical results is less visible in higher, than in lower temperature range.

\section{Conclusions}

Due to doping of the Ag nanoparticles into the porous glass, obtained with the sol-gel technique, the reduction of the sizes of the pores can be observed. Doping the sol-gel glass with Ag NPs probably affects the PALS measurement by interaction with free positrons (trapping in metallic centers before positronium formation) and positronium (quenching). Before heating, the nanoparti- 


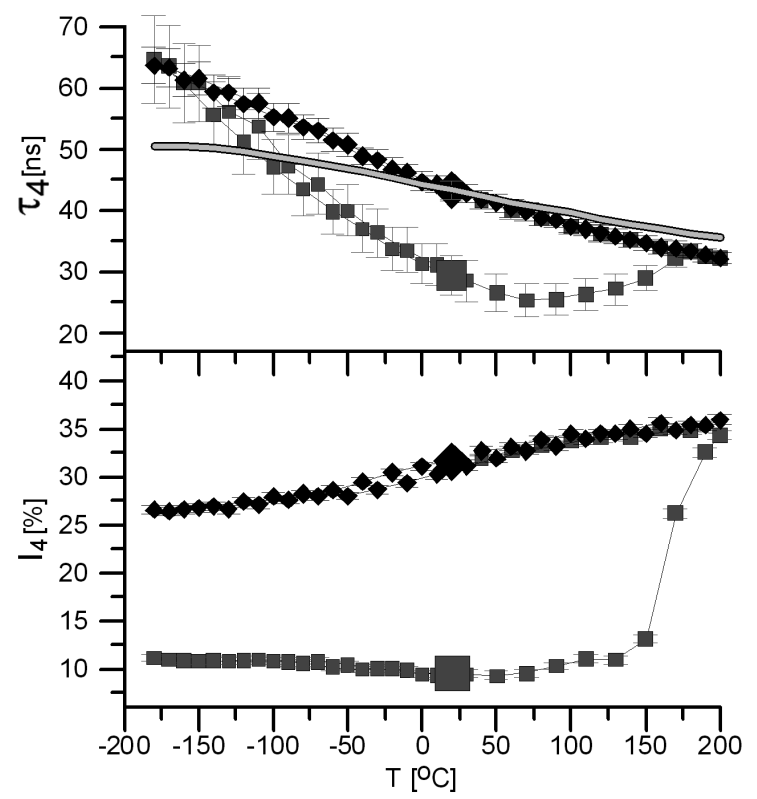

Fig. 3. The $o$-Ps lifetime and intensity of longest-lived component as a function of temperature for glass doped with the Ag nanoparticles. Symbols denote: squares - the first measurement cycle, diamonds - the second measurement (after heating the sample up to $200{ }^{\circ} \mathrm{C}$ and recooling to the room temperature). Initial values are highlighted with enlarged points. Fitted curve shows the approximation of the ETE model for the second measurement cycle.

cles of the size: $10-20 \mathrm{~nm}$ are distributed throughout the material. The structure of such material is unstable due to temperature change. Heating up to $200{ }^{\circ} \mathrm{C}$ resulted in Ag NPs clusters formation and decrease of nanoparticle influence on the intensity value of the fourth component. All these effects can be observed in the PALS measurement.

\section{Acknowledgments}

Authors want to thank Prof. S.V. Stepanov for valuable discussion.

\section{References}

[1] R. Zaleski, J. Goworek, A. Borówka, A. Kierys, M. Wiśniewski, in: Characterisation of Porous Solids VIII, Eds. N. Seaton, F.R. Reinoso, P. Llewellyn, S. Kaskel, RSC Publishing, Cambridge, p. 400.

[2] C.G. Fisher, M.H. Webber, C.L. Wang, S.P. McNeil, K.G. Lynn, Phys. Rev. B 71, 180102 (2005).

[3] S. Kunishige, M. Koshimizu, K. Asai, Radiat. Phys. Chem. 78, 1088 (2009).

[4] S. Thraenert, E.M. Hassan, D. Enke, D. Fuerst, R. Krause-Rehberg, Phys. Status Solidi C 4, 3819 (2007).

[5] R. Zaleski, J. Wawryszczuk, J. Goworek, A. Borówka, T. Goworek, J. Coll. Interface Sci. 262, 466 (2003).

[6] T. Goworek, K. Ciesielski, B. Jasińska, J. Wawryszczuk, Chem. Phys. Lett. 272, 91 (1997).

[7] R. Reisfeld, T. Saraidarov, G. Panzer, V. Levchenko, M. Gaft, Opt. Mater. 34, 351 (2011).

[8] T. Saraidarov, V. Levchenko, R. Reisfeld, Phys. Status Solidi C 7, 2648 (2010).

[9] J. Kansy, Nucl. Instr. Methods Phys. Res. A 374, 235 (1996).

[10] A. Shukla, M. Peter, L. Hoffmann, Nucl. Instrum. Methods Phys. Res. A 335, 310 (1993).

[11] R. Zaleski, EELViS, http://eelvis.sourceforge. net, Accessed: 18 Jan. 2010.

[12] B. Sláviková, M. Miklošovičová, E. Illeková, V. Majernik, O. Šauša, J. Krištiak, K. Jesenák, Mater. Sci. Forum 733, 107 (2013).

[13] B. Jasińska, M. Śniegocka, R. Reisfeld, E. Zigansky, Nukleonika 58, 241 (2013).

[14] M. Śniegocka, Ph.D. Thesis, Maria Curie-Skłodowska University, Lublin 2009.

[15] M. Gorgol, R. Zaleski, A. Kierys, Nukleonika 58, 229 (2013). 